\title{
President's Message: Rebuilding Our Identity, Together
}

ITAL is the official journal of LITA (Library and Information Technology Association), and if you are a reader of the ITAL journal, it is highly likely that you are a member of LITA and/or one who is deeply interested in library technology. It is my pleasure to write this column to update all of you about the exciting discussion that is currently underway in LITA and two other ALA divisions, ALCTS (Association for Library Collections and Technical Services), and LLAMA (Library Leadership and Management Association).

As many of you know, LITA began discussing the potential merger with two other ALA divisions, ALCTS and LLAMA, last year. ${ }^{1}$ What initially prompted the discussion was the prospect of continuing budget deficits in all three divisions. But the resulting conversation has proved that financial viability is not the entire story of the change that we want to bring about.

At the 2018 ALA Annual Conference in New Orleans, the three Boards of LITA, ALCTS, and LLAMA held a joint meeting open to members and non-members alike to solicit and share our collective thoughts, suggestions, concerns, and hopes about the potential three-division realignment. At this meeting attended by approximately 75 people, participants expressed their support for creating a new division with the following key elements.

- Retain and build upon the best elements of each division.

- Embrace the breakdown of silos and positive risk-taking to better collaborate and move our profession forward.

- Build a strong culture of innovation, energy, and inspiration.

- Be more transparent, responsive, agile, and less bureaucratic

- Excel in diversity, equity, and inclusion.

- Support members in all stages of their careers, those with the least means to travel for in-person participation, in particular.

- Provide member-driven interactions and good value for the membership fee.

These ideas have made it clear that members of all three divisions see the goal of realignment as something much more fundamental than financial sustainability. They have validated the shared belief among the LITA, ALCTS, and LLAMA Boards that the ultimate goal of realignment is to create a division that better serves and benefits members, not to simply recover the division's financial health.

While the criteria for the success of a new combined division received almost unanimous endorsement at the meeting, opinions about how to realize such success varied. There were understandable concerns associated with combining three small-sized associations into one large one. For example, how will we reconcile three distinctly different cultures in LITA, ALCTS, and LLAMA? How will the new association ensure itself to be more transparent, responsive, and

Bohyun Kim (bohyun.kim.ois@gmail.com) is LITA President 2018-19 and Chief Technology Officer \& Associate Professor, University of Rhode Island Libraries, Kingston, RI. 
nimble than the individual divisions prior to the merger? Could the larger size of the new division make it more difficult for small groups with special interests to get needed support for their programs? Many requested that the leadership of the three divisions provide more specific vision and details.

As a group, the leaders of LITA, ALCTS, and LLAMA are committed to hashing out those details. With the aim of providing fuller information about what the new division would look like at the 2019 Midwinter Conference, we have already formed working groups, one for finances and the other for communication and are currently working to create two more on operations and activities. These four teams will work closely together with the current leadership of LITA, ALCTS, and LLAMA, to prepare the most important information about the proposed new division, so that the boards and the members of three divisions can review and provide feedback for needed adjustments. Our goal is to present essential information that will allow the members to vote with confidence on the proposal to form one new division on the ALA ballot in the Spring of 2019. If the membership vote passes, then we will be taking the proposal to the ALA Committee on Organization for finalization.

On this occasion, I would also like to bring to everyone's attention to an inherent tension between the two ideas that many of us hold as association members regarding alignment. One is that more member involvement in determining alignment-related details at an early stage is essential to the success of the new division. The other is that we can decide whether we will support the new division or not, only after the leadership first presents us with a clear, specific, and detailed picture of what the new division will look like. The problem is that we cannot have both at the same time. As members, if we want to be involved at an early stage of reorganization, we will have to accept that there will be no ready-made set of clear and specific details about the division waiting for us to simply say yes or no. We will be required to work through our collective ideas to decide on those details ourselves. It will be a messy, iterative, and somewhat confusing process for all of us.

There is no doubt that this will be hard work for both the LITA leadership and LITA members. But it is also an amazing opportunity. Imagine a new division, where (a) innovative ideas and projects are shared and tested through open conversation and collaboration among library professionals in a variety of functional areas such as systems and technology, metadata and cataloging, and management and administration, (b) frank and inspiring dialogues take place between front-line librarians and administrators about vexing issues and exciting challenges, and (c) new librarians learn the ropes, are supported throughout their careers going through changes in their responsibilities as well as areas of specialization, are mentored to be future leaders, and get to develop the next generation of leaders as they themselves achieve their goals. Furthermore, I believe that the process of building this kind of new association from the ground up will be a truly rewarding experience. We had an opportunity to discuss and share our collective hope and vision for the new division at the joint meeting, and that vision is an inspiring one: a division that is member-driven, nimble and responsive, transparent and inclusive, and not afraid to take risks. Can we create a new association that breaks down our own silos and builds bridges for better communication and collaboration to move our profession forward? 
My hope is that we can model and embody the change we want to see, starting in the reorganization process itself. If we want to build a new association that is inclusive, transparent, and nimble, we should be able to build such an association in precisely that manner: inclusively, transparently, and nimbly. If we are successful, our identity as members of this new division will be rebuilt as the very spirit and energy of continuing innovation, experimentation, and collaboration across different functional silos of librarianship, rather than as what we have in our job titles.

Many LITA members and ITAL readers are leaders in their field and care deeply about the continued success and innovation of LITA and ITAL. I would like to invite all of you to participate in this effort of three-division alignment and to inform and lead our way together. While the boards of three divisions are working on the proposal, there will be multiple calls for member participation. Keep your eye out for new updates that will be posted in the ALA Connect community, "ALCTS/LLAMA/LITA Alignment Discussion" at https://connect.ala.org/communities/community-home?CommunityKey=047c1c0e-17b9-45b6a8f6-3c18dc0023f5. All information in this group site is viewable to the public. LITA, ALCTS, and LLAMA members can also join the group, post suggestions and feedback, and subscribe to updates. Where would you like LITA to be next year, and the year after? Let us take LITA there, together.

\section{ENDNOTE}

${ }^{1}$ Andromeda Yelton, "President's Message," Information Technology and Libraries 37, no. 1 (March 19, 2018): 2-3, https://doi.org/10.6017/ital.v37i1.10386. 